\title{
Laser Cyclophotocoagulation Enhances the Regulative Capacity of Retinal Vessels in Glaucoma
}

\author{
Stephan Kremmer ${ }^{*}, 1,2$, Gerasimos Anastassiou ${ }^{1,2}$, Maurice Schallenberg ${ }^{2}$, Klaus-Peter Steuhl ${ }^{2}$, \\ W. Vilser ${ }^{3,4}$ and J. Michael Selbach ${ }^{1,2}$ \\ ${ }^{I}$ Department of Ophthalmology, Evangelische Kliniken Gelsenkirchen, Munckelstr. 27, 45879 Gelsenkirchen, Germany \\ ${ }^{2}$ Department of Ophthalmology, University of Duisburg-Essen, Hufelandstr. 55, 45122 Essen, Germany \\ ${ }^{3}$ Institut of Biomedical Engineering and Informatics, Ilmenau University of Technology, Ilmenau, Gustav-Kirchhoff- \\ Str.2, Germany \\ ${ }^{4}$ Imedos Systems UG, Jena, Am Naßtal 4, Germany
}

\begin{abstract}
Purpose: To determine the effects of laser surgical IOP reduction by means of transscleral cyclophotocoagulation (CPC) on retinal blood flow parameters in glaucoma patients using Dynamic Vessel Analysis (DVA).

Materials and Methodology: 26 patients (average age: 70 years) with a long history of primary open angle glaucoma underwent CPC. The effect on the reactive capacity of retinal vessels was assessed before and 6-8 weeks after CPC by means of the Dynamic Vessel Analyzer (DVA) using flicker light provocation.
\end{abstract}

Results: In our group of POAG patients, IOP was significantly reduced about approximately $20 \%$ by CPC while systemic blood pressure and heart rate were not changed.

The most obvious differences between the pre- and postoperative DVA measurements could be observed in the maximal dilation of the retinal arteries which increased from $0.75 \%(+/-0.6)$ to $3.17 \%(+/-0.5)$ with an average increase of $2.4 \%$ $(\mathrm{p}<0.01)$. In addition, the ability of the arteries for constriction improved significantly $(\mathrm{p}<0.05)$ while the dynamic responses of the veins and the initial baseline values (MU) of the vessel diameters did not change.

Conclusions: Our results of DVA measurements after an IOP-lowering laser surgical intervention (CPC) reveal a significant recovery of the regulative capacity of retinal arteries in glaucoma patients that has up to now neither been properly documented nor appreciated. Future studies with long-term follow-up must determine the clinical importance of these findings for the treatment of glaucoma patients.

Keywords: Cyclophotocoagulation (CPC), glaucoma, IOP, retinal vessel autoregulation, dynamic vessel analyzer (DVA), vascular dysregulation.

\section{INTRODUCTION}

Since the days of Albrecht von Graefe, treating glaucoma has been synonymous with lowering the intraocular pressure (IOP). The means to achieve IOP lowering are either pharmacological or surgical. Glaucoma surgery these days offers a wide variety of options ranging from laser treatment to penetrating and non-penetrating procedures. While some measures are intended to improve the outflow of aqueous humor, curbing the production of this substance by treating the ciliary body is another way to reduce IOP.

Cyclophotocoagulation (CPC) is a proven method to treat the ciliary body and can be administered either transsclerally or endoscopically. Transscleral CPC is well suited for

*Address correspondence to this author at the Department of Ophthalmology, Evangelische Kliniken Gelsenkirchen, Munckelstr. 27, 45879 Gelsenkirchen, Germany; Tel: 0049 - 209 - 37261; Fax: 0049 - 209-378555;

E-mail: stephan.kremmer@arcor.de primary glaucoma treatment as Grueb et al. have demonstrated [1]. It is therefore justified to commence surgical treatment by CPC as an alternative to other more invasive procedures like, for instance, trabeculectomy.

An elevated IOP is probably the most important risk factor for glaucomatous damage to the optic nerve, but for many patients IOP is far from being the sole pathogenetic entitiy. Among the other non IOP-related risk factors that have been identified as causative agents for glaucomatous damage, the vascular abnormalities stand out. Patients with normal-tension glaucoma (NTG) are suffering to a high degree from low arterial blood pressure, fluctuations and nocturnal dips in blood pressure and disturbances of the blood vessels' autoregulation. These symptoms are frequently part of a pathological process that Flammer named "vascular dysregulations" [2].

Improving autoregulation in the retinal blood vessels is presumed to add to the therapeutic benefit of IOP lowering 
[3]. It was the aim of our study to evaluate the retinal vessels' response to a laser surgically-reduced IOP level and to find out whether the lower IOP improves the responsiveness of retinal arteries and veins in patients who had not been successfully treated with glaucoma medications.

Over the last couple of years Dynamic Vessel Analysis (DVA) emerged as a method to gather objective and quantitative data on the efficiency and function (or dysfunction) of retinal vessels. The test uses a device called Retinal Vessel Analyzer (RVA) which provides a fast and non-invasive evaluation of changes in retinal blood vessel diameter [4,5].

Flicker-evoked dilation of retinal arterioles measured by the DVA has proven to be a parameter that is suitable as a functional parameter of the regulation ability of retinal arteries [6]. Nguyen et al. have demonstrated the high reproducibility of DVA for repeated measures over a short period of time in a study group of 33 healthy subjects. The authors reached the conclusion that such measurements may allow non-invasive quantification of endothelial function to study its association with systemic and ocular diseases [7]. A general overview of the use of DVA in research and in a clinical setting and informed guidelines for its use have recently been given by Garhofer et al. [8].

It was the goal of our study to find out whether transscleral CPC has an effect on the reaction of retinal blood vessels.

\section{MATERIALS AND METHODOLOGY}

Our study group consisted of 26 POAG (primary open angle glaucoma) patients. All patients underwent medical therapy for many years. Current medication of the patients consisted of various antiglaucomatous eye drops (average: 2.6 medications).

CPC was performed because of insufficient IOP regulation despite the medication, eye drop intolerance, or compliance problems. Patients were recruited consecutively from our glaucoma service and gave their informed consent. The study was performed in accordance to the declaration of Helsinki on good medical practice.

Exclusion criteria for this study were all kinds of previous glaucoma surgery, other eye diseases (such as macular degeneration; only mild cataract allowed), diabetes and arterial hypertension (defined as a median blood pressure of more than $160 / 90 \mathrm{~mm} \mathrm{Hg}$ ). Therefore none of our patients had any systemic antidiabetic or antihypertensive medication.

Our patients were treated by transscleral cyclophotocoagulation using an IRIS Medical OcuLight SLx diode laser (Iridex Corporation, California, USA). During each procedure, 20 spots were applied at the inferior 270 degrees of the eye with the tip of the laser device positioned at the limbus. The maximum power output for each spot was $1800-2000$ $\mathrm{mW}$, the duration was $2000 \mathrm{msec}$. As widely recommended [10], the 3- and 9-o'clock positions were avoided.

Our patients underwent Dynamic Vessel Analysis before CPC and at a follow-up between 6 and 8 weeks postoperatively. Anti-glaucomatous medication was identical at both visits. No other eye drops were applied at least 2 weeks before measurement procedure.

Because of the advanced stage of the patients' glaucoma, there was no washout of antiglaucomatous medication before the procedure which also ensured that the measured effect would be independent of any given medical therapy.

The Vessel Analysis with the DVA (Dynamic Vessel Analyzer of Imedos Systems UG, Jena, Germany) is a method to examine retinal vessel parameters noninvasively based on vessel diameter measurements to describe their function (autoregulative vessel responses due to stimulation or provocation of the microcirculation). The DVA was developed to analyze retinal vessels with diameters between 60 and $200 \mu \mathrm{m}$. For the study described here, we used a Dynamic Vessel Analyzer to investigate the arterial and venous flicker response before and after treatment.

The device takes video image sequences of the fundus under green monochromatic light using a special CCD camera fitted to a retinal camera. The images are digitized and analyzed online by a computer which is part of the DVA system. Along each selected vascular segment of up to $2 \mathrm{~mm}$ in length, the vessel's diameter is determined image-byimage in real time. The possible time resolution is 25 measurements per second, the spatial resolution $18 \mu \mathrm{m}$ in the vessel direction and $<1 \mu \mathrm{m}$ in the measurement direction [4].

The measuring principle of the DVA is basically less a direct assessment of the vessel's width but rather a measurement of the diameter of the column consisting of red blood cells streaming in the artery or vein. These blood cells absorb a certain amount of light emitted by a fundus camera and reflected by different retinal layers. Measurement algorithms of the DVA assess vessel diameters from brightness profiles and compensate for disturbances like shadowing structures and reflections on the vessel surface. A properly dilated pupil being essential for the examination's validity, the observer defines a measurement area by dragging a "region of interest" over a chosen segment of an artery and vein. Then the measurements are started by a mouse click. During data acquisition, the diameters of the selected arterial and venous vessel are recorded online. The whole procedure can be watched on a live monitor [5].

During measurements, flicker light stimulation of the retina is performed. The flicker light induces metabolic demand of retinal tissue causing diameter changes of the retinal vessels. This behaviour is based on so called neurovascular coupling and is recorded over time for all segments of the selected vessel. For this the vessel diameter will be recorded over 350s (about 6 minutes) continuously. After recording of the baseline for $50 \mathrm{sec}$ three flickering cycles will follow. These flickering cycles consist of $20 \mathrm{sec}$ flicker followed by a resting period of $80 \mathrm{sec}$. In order to carry out the flickering period a stimulation unit will interrupt the green illumination light in an image synchronic way (one image bright, one image dark, frequency $12.5 \mathrm{~Hz}$, bright/dark contrast 25:1). Measurements are always recorded during the flickering period in the bright image. Healthy vessels react to flickering light with dilation. In the recovery period the diameters constrict to the baseline with an overshooting period (temporary vasoconstriction). The measuring program determines by signal averaging out of 
the 3 measuring cycles (flicker phase and resting period) the mean vessel response to flickering light as a relative value to the baseline. As parameters the maximum flicker dilation and the maximum constriction will be determined. Flickerevoked dilation of retinal arterioles measured by the DVA has proven to be a parameter that is suitable as a functional parameter of the regulation ability of retinal arteries $[6,7]$.

A detailed description of the use of DVA and informed guidelines for its use have recently been given by Garhofer et al. [8]. Our measurements were performed according to this clinical protocol. A muscarinic antagonist was used for pupil dilation, vasoactive drugs such as caffeine or nicotine were avoided before measuring.

After the measurement procedure, the software of the DVA provides several vessel parameters:

- $\quad$ initial baseline value for the width of artery and vein given in measuring units (MU); $1 \mathrm{MU}=1 \mu \mathrm{m}$ if the eye is fulfilling the conditions of the Gullstrand normal eye. For many eyes this is not the case and interindividual aberrations must be expected. Therefore, no absolute values are given but the term measuring units (MU) is introduced allowing an intraindividual comparison.

- $\quad$ maximum dilation (as \% of the initial baseline) of artery and vein during flicker stimulation

- $\quad$ maximum constriction after flicker stimulation

- peak amplitude (difference between dilation and constriction of artery)

For statistical analysis a t-test was used. $\mathrm{P}<0.05$ was considered the level of significance. Results are given as mean $+/$ - standard error of the mean.

\section{RESULTS}

Our study group consisted of 26 POAG (primary open angle glaucoma) patients (15 female, 11 male) with an average age of 70.0 years $(+/-2.0)$. Mean defect of visual field was 9.8 and mean cup-to-disc ratio 0.78 .

In these patients with long existing, sometimes refractory glaucoma, eye drop intolerance, and compliance problems, CPC proved to be a safe and effective procedure to lower IOP. At the time of re-examination (4-6 weeks after laser surgery), the average pre-surgery IOP of $21.1(+/-0.7) \mathrm{mm}$ $\mathrm{Hg}$ had been reduced to $16.7(+/-0.6) \mathrm{mm} \mathrm{Hg}$. This is a mean reduction of $4.4 \mathrm{~mm} \mathrm{Hg}$ or of $20.4 \%(\mathrm{p}<0.01)$. None of the patients showed any signs of ocular inflammation as anterior chamber cells or flare in the slit-lamp examination.

Mean systolic blood pressure at the time of the DVA measurement was $140.9(+/-3.7) \mathrm{mm} \mathrm{Hg}$ at the first examination before the laser procedure and $142.8(+/-3.7)$ $\mathrm{mm} \mathrm{Hg}$ at the second examination after several weeks. The difference was not significant. Mean pre-operative diastolic blood pressure was $78.1(+/-2.0) \mathrm{mm} \mathrm{Hg}$, post-operative diastolic pressure was 79.3 (+/-) $1.9 \mathrm{~mm} \mathrm{Hg}$ (not significant).

The initial baseline values of arteries and veins (MU) did not deviate significantly before and after CPC treatment (artery pre-CPC $110.9+/-4.5 \mathrm{MU}$, post-CPC $109.6+/-3.5$ MU; vein pre-CPC 138.5 +/- 3.0 MU, post-CPC 141.1 +/4.3 MU; not significant).
Measuring the retinal vessels' response to the flicker light using Dynamic Vessel Analysis, the maximum dilation and peak amplitude of the retinal artery chosen for DVA evaluation increased significantly (Fig. 1a, b). The maximal dilation increased from $0.75 \%+/-0.56$ before CPC to 3.17 $\%+/-0.48$ after CPC, an average increase of $2.42 \%$ $(\mathrm{p}<0.01)$.

Accordingly, the arteries' ability for constriction changed significantly $(\mathrm{p}<0.05)$ from $-0.21 \%$ to $1.70 \%$. These results can be seen in Fig. (1c).

Non-significant changes were measured by the DVA for the maximum dilation of retinal veins (Fig. 1d).

\section{DISCUSSION}

In our group of patients with primary open angle glaucoma, DVA has demonstrated a significant response of the retinal vessels' function following IOP-lowering by laser-surgical procedures (cyclophotocoagulation), a response that points towards an improved autoregulation of these blood vessels.

In another study with glaucoma patients we could not find a major impact of topical glaucoma therapy with latanoprost on retinal haemodynamics despite pronounced IOP lowering of about $25 \%$. There was no significant change in retinal vessel diameter or regulative capacity to increased demand provoked by flicker light in these patients (*** Influence of Latanoprost on Retinal Microcirculation in Glaucoma, under review).

Over the past few years, Dynamic Vessel Analysis (DVA) has emerged as a helpful tool in measuring retinal circulation and observing disturbances in the regulative capacity of these vessels. Dynamic Vessel Analysis is considered to have a high potential for clinical interdisciplinary cooperation in the field of systemic vessel diseases but also for ophthalmological diseases like glaucoma, vascular occlusive disease and diabetic retinopathy $[9,10]$.

Some studies using DVA pointed out impaired vessel response to stimulation as a possible underlying cause for well-known clinical entities like retinopathy. It is now an established fact that flicker responses of retinal vessels are abnormally reduced in diabetic patients [11]. Other studies using DVA have examined alterations in retinal blood flow and autoregulation in patients with systemic hypertension [6]. In elderly patients DVA has been used to study the effect of the aging blood vessels [12]; the technology has also been applied to patients with cardiovascular risk factors [13].

The fact that retinal vessels in glaucoma are displaying abnormalities in their autoregulation has been established in an earlier study using DVA [14]. The Austrian group examined 31 patients with early stage glaucoma and 31 ageand sex- matched healthy volunteers. Flicker-induced vasodilatation in retinal veins was significantly diminished in glaucoma patients as compared with healthy volunteers. In retinal arteries, no significant difference in flicker response was noticed between the two groups. The authors concluded 
a

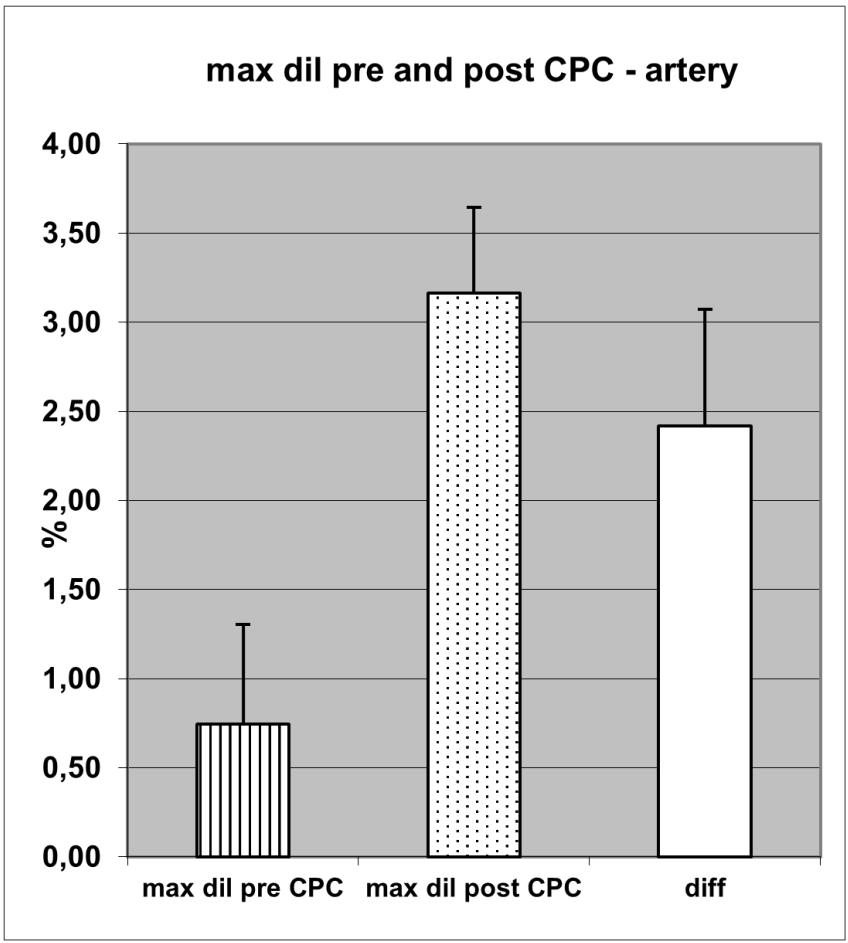

C

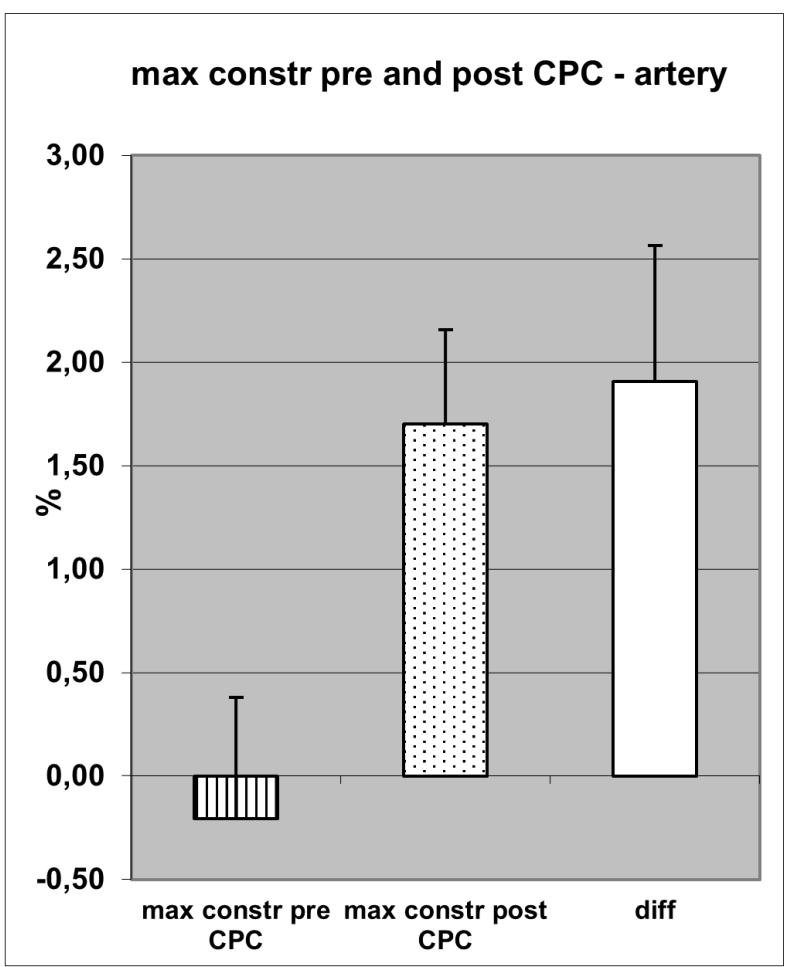

b

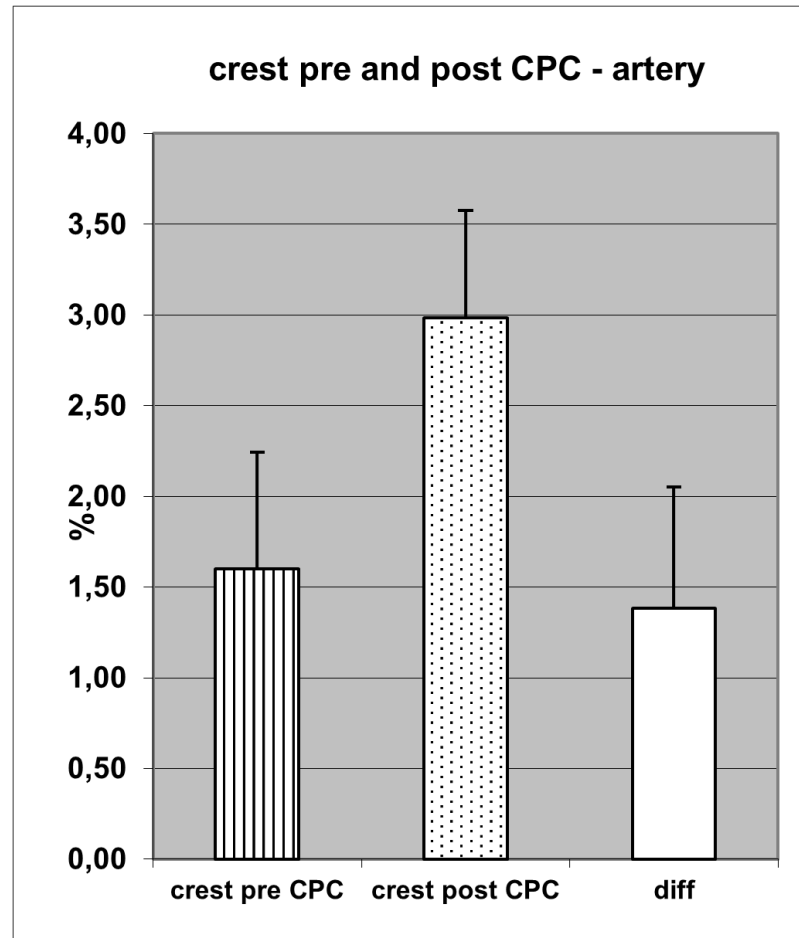

d

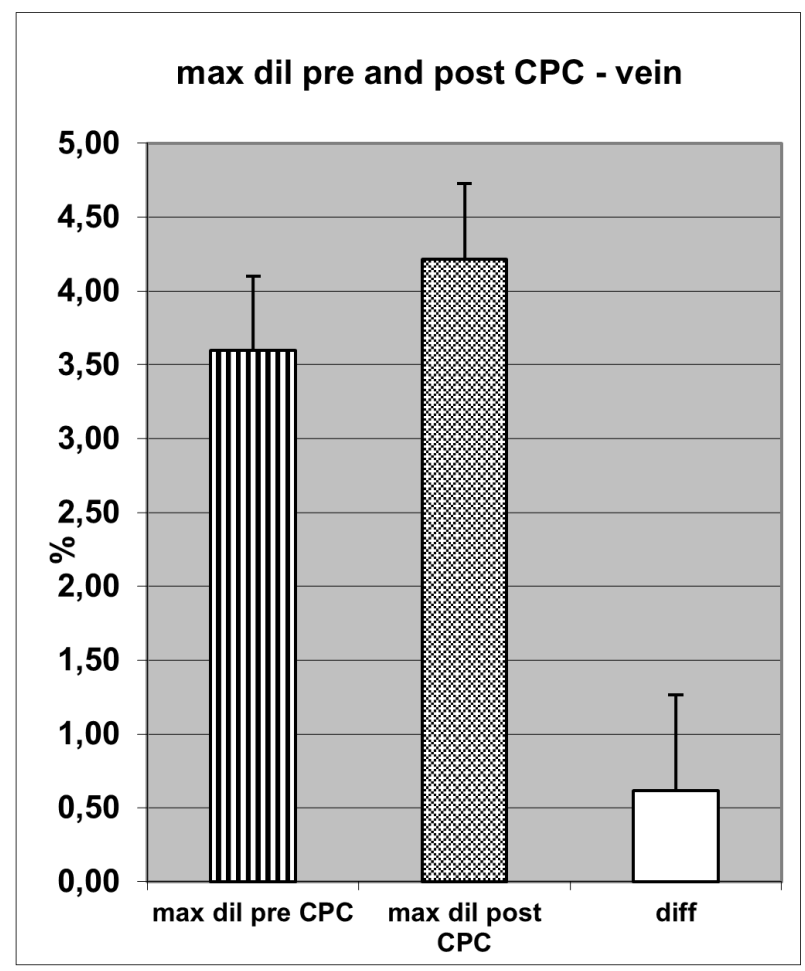

Fig. (1). (a) The average arterial dilation at the end of the flicker stimulus was diminished before (pre) and recovered markedly after (post) cyclophotocoagulation $(\mathrm{p}<0.01)$. (b) The arterial dilation peak maximum during the last $10 \mathrm{sec}$ of the flicker stimulus tended to be lower before (pre) than after (post) cyclophotocoagulation but the increase was not statistically significant (ns). (c) The arterial constriction peak maximum after the end of the flicker stimulus was very low before (pre) and increased significantly after (post) cyclophotocoagulation $(p<0.05)$. (d) The average venous dilation at the end of the flicker stimulus increased only slightly and revealed no significant change between measurements before (pre) and after (post) cyclophotocoagulation (ns). 
that these results indicate impairment in the regulation of retinal vascular tone in patients with early glaucoma, independent of antiglaucoma medication. An improvement in oxygen saturation in the retinal vessels should not be expected to be a result of IOP-lowering surgery, as was demonstrated by Hardarson et al. [15].

In our study, CPC led to a further IOP reduction (after having received medical therapy for years) in the range of $20 \%$ which is quite a satisfactory result in efforts to treat refractory glaucoma. To the best of our knowledge this study is the first to demonstrate a beneficial effect of an IOPlowering surgical intervention on the vascular response of those retinal vessels that had a diminished reaction before surgery despite an often long history of topical antiglaucomatous therapy.

DVA thus becomes the first diagnostic method to objectively prove the effect of IOP lowering on the retinal blood flow and, in consequence, for the chance of the ganglion cells to survive a given IOP level.

The ability of DVA to determine the efficacy of a therapeutic approach has been proven by Lanzl et al. [16]: Patients with age-related macular degeneration (AMD) had an altered vascular response at baseline. Three months after a single injection of a VEGF-Inhibitor, these vascular parameters showed a marked improvement.

According to our results DVA is providing evidence that lowering IOP by CPC is a method to increase the regulative capacity and retinal perfusion. Additionally, in glaucoma patients with disease progression despite maximum topical therapy, DVA may provide important information for the indication of laser treatment or surgical therapy.

However, long-time follow-up studies have to show if glaucoma patients have a greater benefit from invasive intraocular pressure lowering therapy by surgery or laser which leads to an improved dilation of retinal vessels on metabolic demand but carrying perhaps the danger of reperfusion damage $[17,18]$ or whether a topical treatment with drugs like latanoprost that lowers the intraocular pressure but does not alter vessel reaction in a statistically significant way is to be preferred.

\section{CONFLICT OF INTEREST}

The authors declare that they have no conflict of interest.

The only exception is Walthard Vilser who is CEO of IMEDOS Systems UG, Germany.

\section{ACKNOWLEDGEMENTS}

Supported by Ernst und Berta Grimmke-Stiftung, Vogelsanger Weg 49, 40470 Düsseldorf, Germany.

\section{REFERENCES}

[1] Grueb M, Rohrbach JM, Bartz-Schmidt KU, et al. Transscleral diode laser cyclophotocoagulation as primary and secondary surgical treatment in primary open-angle and pseudoexfoliatve glaucoma. Long-term clinical outcomes. Graefes Arch Clin Exp Ophthalmol 2006; 244: 1293-9.

[2] Flammer J. To what extent are vascular factors involved in the pathogenesis of glaucoma? In: Kaiser HJ, Flammer J, Hendrickson P, Eds. Ocular blood flow: new insights into the pathogenesis of ocular disease. Basel: Karger 1996; 12-39.

[3] Kremmer S, Selbach JM, Schäfers RF, Philipp T, Steuhl KP. Cardiovascular Risk Profile in the Progression of Glaucomatous Damage [Article in German]. Deutsches Ärzteblatt 2000; 97: A2241-A2245

[4] Nagel N, Vilser W. Autoregulative behavior of retinal arteries and veins during changes of perfusion pressure: a clinical study. Graefe's Arch Clin Exp Ophthalmol 2004; 242: 19-17.

[5] Seifert BU, Vilser W. Retinal Vessel Analyzer (RVA) - Design and Function. Biomed Tech Suppl 2002; 1: 47.

[6] Nagel E, Vilser W, Fink A. Variance of retinal vessel diameter response to flicker light [Article in German]. A methodical clinical study. Ophthalmologe 2006; 103: 114-19.

[7] Nguyen TT, Kreis AJ, Kawasaki R, et al. Reproducibility of the retinal vascular response to flicker light in Asians. Curr Eye Res 2009; 12: 1082-8.

[8] Garhofer G, Bek T, Boehm A, et al. Use of the retinal vessel analyzer in ocular blood flow research. Acta Ophthalmol 2010; 88: 717-22.

[9] Heitmar R, Blann A, Cubbidge RP, et al. Continuous retinal vessel diameter measurements - the future of retinal vessel assessment? Invest Ophthalmol Vis Sci 2010; 51: 5833-9.

[10] Lin SC. Endoscopic and transscleral cyclophotocoagulation for the treatment of refractory glaucoma. J Glaucoma 2008; 17: 238-47.

[11] Schiel R, Vilser W, Kovar F, et al. Retinal vessel response to flicker light in children and adolescents with type 1 diabetes mellitus and overweight or obesity. Diabetes Res Clin Pract 2009; 83: 358-64.

[12] Kotliar KE, Mücke B, Vilser W, et al. Effect of aging on retinal artery blood column diameter measured along the vessel axis. Invest Ophthalmol Vis Sci 2008; 49: 2094-102.

[13] Reimann M, Prieur S, Lippold B. Retinal Vessel Analysis in hypercholesterolemic patients before and after LDL apheresis. Atheroscler Suppl 2009; 10: 39-43.

[14] Garhofer G, Zawinka C, Resch H. Response of retinal vessel diameters to flicker stimulation in patients with early open angle glaucoma. J Glaucoma 2004; 13: 340-44.

[15] Hardarson SH, Gottfredsdottir MS, Halldorsson GH, et al. Glaucoma filtration surgery and retinal oxygen saturation. Invest Ophthalmol Vis Sci 2009; 50: 5247-50.

[16] Lanzl I, Seidova SF, Maier M, et al. Dynamic retinal vessel response to flicker in age-related macular degeneration patients before and after vascular endothelial growth factor inhibitor injection. Acta Ophthalmol 2010; 22: 1-8.

[17] Flammer J, Mozaffarieh M. Autoregulation, a balancing act between supply and demand. Can J Ophthalmol 2008; 43: 317-21.

[18] Konieczka K, Fränkl S, Todorova MG, Henrich PB. Unstable Oxygen Supply and Glaucoma. Klin Monbl Augenheilkd 2014; 231: $121-6$ 